\title{
Macroscopic Quantum Coherence in a repulsive Bose-Einstein condensate.
}

\author{
A. Montina and F.T. Arecchi \\ Dipartimento di Fisica, Università di Firenze, \\ Istituto Nazionale di Ottica Applicata, Largo E. Fermi 6,50125 Firenze, Italy.
}

(Dated: October 24, 2018)

\begin{abstract}
We consider a Bose-Einstein bicondensate (BEC) of ${ }^{87} R b$, trapped in two different internal levels, in a situation where the density undergoes a symmetry breaking in momentum space. This occurs for a suitable number of condensed atoms within a double well dispersion curve, obtained by Raman coupling two internal states with two tilted and detuned light fields. Evidence of bistability results from the Gross-Pitaevskii equation. By second quantization, we evaluate the tunneling rate between the two asymmetric states; the effects of losses on coherence are also considered.
\end{abstract}

The transition from a superfluid to a Mott insulator has been recently demonstrated for a Bose-Einstein condensate (BEC), made of atoms with repulsive mutual interactions in a lattice potential [1]. Such a phenomenon goes beyond the mean field approximation and its explanation requires to take into account the quantum fluctuations of the matter field. When the quantum tunneling between adjacent sites dominates the interaction energy, the probability distribution for the atomic occupation of a single site is Poissonian. In the opposite case the minimum energy is obtained reducing the quantum fluctuation of the local occupation number.

In a previous paper we have studied a similar problem, but with only two wells and an attractive interaction [2]; In this case the opposite effect occurs, that is, raising the atom number, i.e. the interaction energy, the minimum energy state is obtained increasing the atomic fluctuation in each site. This is indirectly demonstrated by the numerical observation of the symmetry breaking at a critical number of atoms. Approaching the threshold value, the quantum fluctuations increase, whereas above that value they blow up and a new minimum quantum state appears. The associated probability distribution of the condensate barycenter displays two peaks, that can be considered as the dead and alive states of a Schrödinger cat (SC), whose coherent superposition is called Macroscopic Quantum Coherence (MQC). This term was introduced to describe the coherent superposition of two macroscopically distinct quantum states that differ for the value of a collective variable [3]. The phenomenon is observable only for attractive interactions, that tend to cluster the atoms in one of the two wells. By contrast, a repulsive interaction tends to reduce the quantum fluctuations and to distribute the same number of atoms in each well, as observed in Ref. 1. MQC has been observed with trapped ions [4] and microwave fields in high-Q cavity [5].

In this work, we discuss the feasibility of MQC in a BEC of mutually repulsive atoms. A repulsive interaction acts in the momentum space as an attractive interaction, therefore we expect that for a double well dispersion curve the symmetry breaking occurs in the reciprocal space. Such a dispersion curve can be obtained by two detuned and tilted light fields, that transfer a net momentum to atoms as they jump from an internal state to another. We study the problem by finding the stationary solutions of two coupled Gross-Pitaevskii equations (GP) discretized over a space lattice. The system undergoes symmetry breaking in momentum space for a suitable number of atoms and two new stationary states are created. We then introduce a quantum two mode model, with the two modes chosen in such a way as to reproduce the stationary solutions of GP, and evaluate the quantum fluctuation-mediated tunneling rate between the two asymmetrical states. If the coupling with the environment is negligible, MQC occurs between these states. A Raman scheme for creating a superposition state with two $R b$ condensates in different internal quantum levels has already been discussed in Refs. 6, 7; however both papers limit themselves to co-propagating light beams, and this implies applicability problems, as discussed in Ref. 2.

Here we refer to ${ }^{87} R b$ atoms, but our numerical results apply also to ${ }^{23} \mathrm{Na}$, if some parameters are rescaled. In a previous work [8] we considered atoms in two different hyperfine levels $\left(F=1, m_{F}=-1\right.$ and $\left.F=2, m_{F}=1\right)$, however the associated depletion rate [9] is too high for our purposes. Here we consider condensate atoms that are optically trapped in the two Zeeman levels $F=1$, $m_{F}=-1$ and $F=1, m_{F}=1$. An all optical condensation has been reported in Ref. 10, alternatively, the condensate can be created with a magnetic confinement and transferred into an optical trap [11. A homogeneous magnetic field has to be applied to remove the energy degeneracy. These levels are quasi-resonantly coupled by means of two Raman fields $L$ and $R$. We call $\psi_{0}$ and $\psi_{1}$ the fields associated with the $m_{F}=-1$ and $m_{F}=1 \mathrm{lev-}$ els, respectively. Furthermore we call $\psi_{2}$ the upper state of the $D_{1}$ transition.

The starting equations are

$$
i \hbar \dot{\psi}_{0,1}=\left(H_{0,1}-\hbar \omega_{0,1}\right) \psi_{0,1}+\hbar E_{L, R}(t) e^{-i\left(\vec{k}_{L, R} \cdot \vec{x}-\omega_{L, R} t\right)} \psi_{2}
$$

$$
i \hbar \dot{\psi}_{2}=\hbar \omega_{2} \psi_{2}+\left[\hbar E_{L}^{*}(t) e^{i\left(\vec{k}_{L} \cdot \vec{x}-\omega_{L} t\right)} \psi_{0}+(L \leftrightarrow R) \psi_{1}\right]
$$

where $H_{0}=H_{l}+g_{00}\left|\psi_{0}\right|^{2}+g_{01}\left|\psi_{1}\right|^{2}, H_{1}=H_{l}+g_{11}\left|\psi_{1}\right|^{2}+$ $g_{10}\left|\psi_{0}\right|^{2}$ and $g_{i j}=4 \pi \hbar^{2} a_{i j} / m . a_{i j} \sim 5.5 \mathrm{~nm}$ [12] are the 
s-wave scattering lengths between atoms in $i$ and $j$ levels. We have called $H_{l}=-\left(\hbar^{2} / 2 m\right) \nabla^{2}+V$ the one atom term of the Hamiltonian, where $V$ is the trapping potential. The field amplitudes $E_{L, R}$ are rescaled in order to be expressed in frequency units. They are thus the Rabi frequencies of the one-photon transition. $\hbar \omega_{2}$ is the energy of the upper state of the one photon transition; $\hbar \omega_{0,1}$ are the energies of the $m_{F}=-1,1$ levels, respectively. We set $\omega_{0}=0$. In the adiabatic approximation, $\psi_{2}$ can be expressed in terms of $\psi_{1}$ and $\psi_{0}$ as $\psi_{2}=-\left[E_{L}^{*} e^{i\left(\vec{k}_{L} \cdot \vec{x}-\omega_{L} t\right)} \psi_{0}+E_{R}^{*} e^{i\left(\vec{k}_{R} \cdot \vec{x}-\omega_{R} t\right)} \psi_{1}\right] / \Delta$, where $\Delta=\omega_{2}-\omega_{L}$. Thus we have two closed equations for $\psi_{0}$ and $\psi_{1}$.

We introduce the gauge transformation

$$
\tilde{\psi}_{0,1}=e^{-i \int \frac{\left|E_{L}\right|^{2}+\left|E_{R}\right|^{2}}{\Delta} d t} e^{ \pm i\left(\frac{\vec{k}_{d}}{2} \cdot \vec{x}-\frac{\omega_{d}}{2} t \pm \frac{\hbar \vec{k}_{d}^{2}}{8 m} t\right)} \psi_{0,1},
$$

where $k_{d}=\vec{k}_{L}-\vec{k}_{R}$ and $\omega_{d}=\omega_{L}-\omega_{R}$. As a result, the equations of motions become

$$
i \hbar \dot{\tilde{\psi}}_{0,1}=\left(H_{0,1} \mp \frac{\hbar \delta}{2}\right) \tilde{\psi}_{0,1}-\hbar \Omega \tilde{\psi}_{1,0} \pm \frac{i \hbar^{2} \vec{k}_{d} \cdot \vec{\nabla}}{2 m} \tilde{\psi}_{0,1}
$$

Here, $\Omega \equiv \frac{E_{L} E_{R}^{*}}{\Delta}$ is the two photon Rabi frequency, taken for simplicity as time independent and real, and the frequency $\delta$ is given by $\delta=\omega_{1}-\omega_{d}+\left(\left|E_{L}\right|^{2}-\left|E_{R}\right|^{2}\right) / \Delta$. We assume $\left|E_{L}\right|^{2}=\left|E_{R}\right|^{2}$ and $\omega_{d}=\omega_{1}$, hence $\delta=0$.

If the number of atoms is sufficiently small, we can neglect the nonlinear terms. Furthermore, let us initially consider a spatially homogeneous condensate (no trap potential). As a consequence, Eqs. (何) reduce to two linear equations with constant coefficients, and the eigenvalue problem in the reciprocal space is ruled by two linear algebraic equations for the transformed fields $\phi_{0}(\vec{k})$ and $\phi_{1}(\vec{k})$. The momenta of the atoms in the two levels $m_{F}=-1$ and $m_{F}=1$ are respectively $\hbar\left(\vec{k}-\vec{k}_{d} / 2\right)$ and $\hbar\left(\vec{k}+\vec{k}_{d} / 2\right)$.

Solving the eigenvalue problem we find the two dispersion curves $\hbar \omega(\vec{k})=\hbar^{2} \vec{k}^{2} /(2 m) \pm\left\{\left[\hbar^{2} \overrightarrow{k_{d}} \vec{k} /(2 m)+\right.\right.$ $\left.\hbar \delta / 2]^{2}+\hbar^{2}|\Omega|^{2}\right\}^{1 / 2}$, plotted in Fig. 偱 for $\Omega=200 s^{-1}$ and $\left|\vec{k}_{d}\right|=2 \pi \cdot 4.5 \cdot 10^{5} \mathrm{~m}^{-1}$. As shown in the figure, the electromagnetic coupling modifies the parabolic dispersion curves associated with the two hyperfine levels, lifting the degeneracy at their intersection point. The energy gap for $\delta=0$ is $2 \hbar|\Omega|$. By varying $\delta$ one can rise or lower the energy separation between the two minima. For $\delta=0$ the two minima have the same energy. Introducing the harmonic trap potential, the ground state has no longer a definite momentum. Furthermore, the two wells of the dispersion curve are equally populated by quantum tunneling (Fig. 1]b, solid line).

When the number of atoms is sufficiently high, a symmetry breaking occurs because of the atomic interactions. We report in Fig. 10 the distributions $\left|\phi_{0}\right|^{2}$ and $\left|\phi_{1}\right|^{2}$ for $\delta=0$ and for three different values of the number of atoms $N . \phi_{0,1}$ are the Fourier transforms of the ground state solution $\left(\tilde{\psi}_{0}, \tilde{\psi}_{1}\right)$ of Eqs. (4) for a spherical trap potential $V$ corresponding to equal longitudinal $\left(\omega_{\perp}\right)$ and

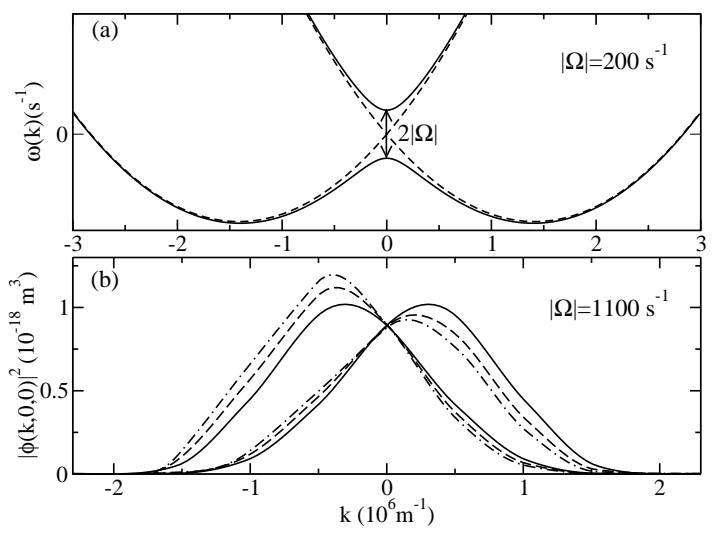

FIG. 1: (a) Dispersion curves of a free atom for $\left|\vec{k}_{d}\right|=2 \pi \cdot 4.5$. $10^{5} \mathrm{~m}^{-1}$ and $\Omega=200 \mathrm{~s}^{-1}$. (b) Density distributions $\left|\phi_{0,1}\right|^{2}$ for $k_{d}=2 \pi \times 4.5 \cdot 10^{5} \mathrm{~m}^{-1}, \Omega=1100 \mathrm{~s}^{-1}, \omega_{\|}=\omega_{\perp}=2 \pi \cdot 100 \mathrm{~s}^{-1}$ and three different boson numbers: $N=1390$ (solid), 1420 (dashed), 1460 (dot-dashed).

radial $\left(\omega_{\|}\right)$trap frequencies. As it results, this interaction clusters the majority of atoms within a single well, thus contrasting the quantum tunneling across the barrier. Due to the geometry of the problem, and taking into account that the scattering lengths $a_{i j}$ are practically equal, there is another ground state which is obtained from that of Fig. 1 $1 \mathrm{~b}$ by inverting the horizontal axis and interchanging $\phi_{0}$ and $\phi_{1}$. Thus we have two stable stationary states with equal energy. The numerical evidence of Fig. 1 $\mathrm{b}$ is also supported by a synthetic variational argument, already exploited in Ref. 2 for $L i$, and based upon a suitable two mode approximation.

The matter field fluctuations allow the passage from one to the other state. If the decoherence is negligible, coherent oscillations between such states can be observed, demonstrating MQC. To evaluate the oscillation frequency we quantize the two mode system, as done in Ref. 2. First of all, we write the classical Hamiltonian corresponding to the equations of motions (伍), taking $g_{00}=g_{11}=g_{01} \equiv g$

$$
\begin{aligned}
& \mathcal{H}=\int\left[\psi_{0}^{*} H_{l} \psi_{0}+\psi_{1}^{*} H_{l} \psi_{1}+\frac{g}{2}\left(\left|\psi_{0}\right|^{2}+\left|\psi_{1}\right|^{2}\right)^{2}+\right. \\
& \left.\frac{i \hbar^{2} \vec{k}_{d}}{2 m}\left(\psi_{0}^{*} \vec{\nabla} \psi_{0}-\psi_{1}^{*} \vec{\nabla} \psi_{1}\right)-\hbar \Omega\left(\psi_{1}^{*} \psi_{0}+\psi_{0}^{*} \psi_{1}\right)\right] d^{3} x
\end{aligned}
$$

(from now on we omit the tilde on the $\psi$ 's, even though we are always in the gauge Eq. (3)). We then introduce the spinorial ground states

$$
\vec{\psi}_{g, 1}(\vec{x}) \equiv\left(\begin{array}{c}
\psi_{0 g}(\vec{x}) \\
\psi_{1 g}(\vec{x})
\end{array}\right) ; \vec{\psi}_{g, 2}(\vec{x}) \equiv\left(\begin{array}{c}
\psi_{1 g}(-\vec{x}) \\
\psi_{0 g}(-\vec{x})
\end{array}\right)
$$

where $\psi_{0 g}$ and $\psi_{1 g}$ are the ground state wave-functions associated with the two internal states. $\vec{\psi}_{g, 2}$ is obtained from $\vec{\psi}_{g, 1}$ by interchanging the spinorial components and inverting the axes. 


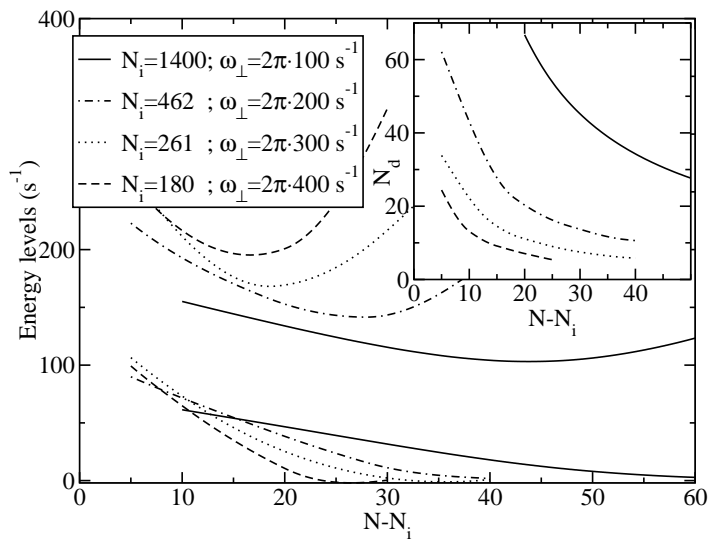

FIG. 2: First and second excited energy levels of the rubidium BEC versus the excess of atoms above the breakup value $N_{i}$ for $\omega_{\|}=2 \pi \cdot 100 s^{-1}$ and some values of $\omega_{\perp}$. In the inset we plot $N_{d}$ for the same values of the trap frequencies.

It is convenient to use the basis vectors $\vec{\psi}_{a}=\vec{\psi}_{g, 1}+\vec{\psi}_{g, 2}$ and $\vec{\psi}_{b}=\vec{\psi}_{g, 1}-\vec{\psi}_{g, 2}$. We write the quantized spinorial field of the bicondensate as $\vec{\psi}(\vec{x})=\hat{a} \vec{\psi}_{a}(\vec{x})+\hat{b} \vec{\psi}_{b}(\vec{x})$, and substitute this expression in the operator version of Eq. (5). Thus, we obtain a reduced Hamiltonian $\mathcal{H}_{\text {red }}$ of the same form found in the attractive case of Ref. 2 . By $\mathcal{H}_{\text {red }}$, we evaluate the difference between the lowest eigenvalues, which provides the tunneling rate. In Fig. 2 we plot the first and second energy level as a function of the number of atoms and for some values of $\omega_{\perp}$. The energy of the ground state is set to zero, so the energy of the first level gives the tunneling frequency. The entanglement between the condensate and the lost atoms induces a decoherence of the superposition. Using the approach of Ref. 2, we find that the coherence is given by $\tilde{C}=e^{-\epsilon M}$, where $M$ is the number of lost atoms and $\epsilon=1-2 \int d^{3} x \psi_{0}^{*}(\vec{x}) \psi_{1}(-\vec{x}) / N$. The quantity $N_{d}=1 / \epsilon$ is the number of atoms which must be lost in order to reduce the coherence by $1 / e$. The inset of Fig. 2 shows how $N_{d}$ scales with $N$. The relevant loss processes are two-body inelastic and threebody collisional decays. In Fig. 3 we report the average three-body and two-body (inset) loss rates, the latter one refers to atoms in the $m=-1$ level. We have used the upper limit of $1.6 \cdot 10^{-16} \mathrm{~cm}^{3} / \mathrm{s}$ for the two-body loss rate coefficient and $5.8 \cdot 10^{-30} \mathrm{~cm}^{6} / \mathrm{s}$ for the three-body processes, both of them measured in Ref. 13 for the trapped Zeeman level $F=1, m=-1$. Two-body decay can occur also by means of collisions between atoms in different Zeeman levels. We suppose that the corresponding loss rate is of the same order of magnitude as the measured value. From Figs. 2, 3 we find that the decoherence effects are negligible during a MQC oscillation period. For high $\omega_{\perp}$ the threshold $N_{i}$ and the loss rates are lower. For $\omega_{\perp}=2 \pi \cdot 200 \div 400 s^{-1}$ the overall loss rate is much smaller than the corresponding tunneling frequencies. Therefore we can observe many oscillations before a single atom is lost. For $\omega_{\perp}=2 \pi \cdot 100 \mathrm{~s}^{-1}$ the loss

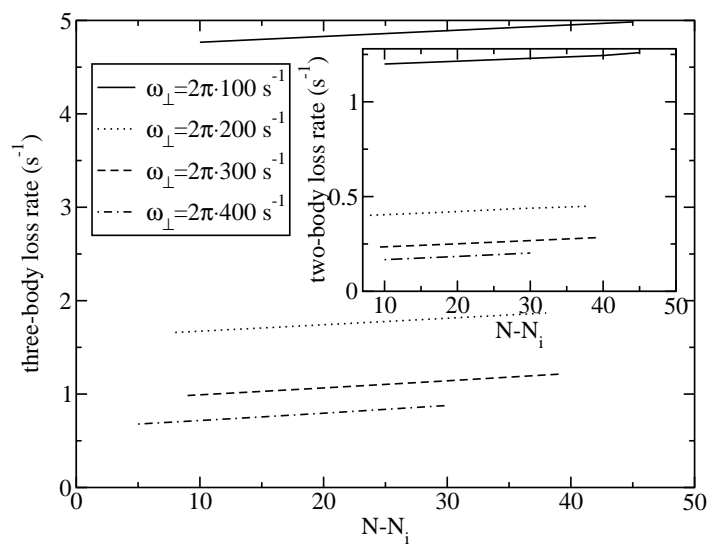

FIG. 3: Three-body and two-body (inset) loss rates. The latter one refers to atoms trapped in the $F=1, m=-1$ level and represents an upper limit 13 .

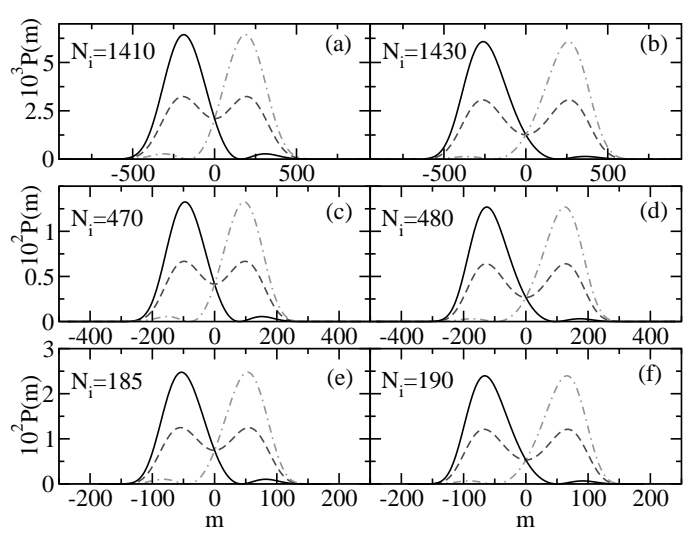

FIG. 4: Distribution $P(m)$ for some parameters. The solid, dashed and dashed-dot lines correspond to $P(m)$ at the initial time, at a quarter of period and half a period, respectively. $\omega_{\perp}$ is (a-b) $2 \pi \cdot 100 s^{-1},(\mathrm{c}-\mathrm{d}) 2 \pi \cdot 200 \mathrm{~s}^{-1}$ and (e-f) $2 \pi \cdot 400 s^{-1}$.

rate is $>5 s^{-1}$. With a tunneling frequency of $8 \mathrm{~Hz}$ also in this case we can observe an oscillation before a single atom is lost. If the loss of atoms does not transfer energy to the trapped atoms, the escape of a few atoms does not reduce the superposition coherence (inset of Fig.2), but modifies slightly the tunneling rate. In Ref. 14 it is shown that inelastic collisional processes induce local variations of the mean-field interparticle interaction and are accompanied by the creation/annihilation of elementary excitations. This phenomenon depends on the density and is completely negligible in our case. Notice that Eqs. (4) are invariant if $N$ is varied by a factor $\alpha$ and the lengths and the energies are multiplied by $\alpha$ and $\alpha^{-2}$, respectively. So for $\omega=\omega_{\perp}=2 \pi \cdot 70 s^{-1}$ the threshold is $N_{i}=1980$. By the two mode model we find that the tunneling frequencies are reduced by nearly a factor 0.5 . However the two-body and three-body decay rates are reduced by the factors 0.25 and 0.125 , respectively.

As we have shown in Ref. 2, the probability distribution $P(m)$ associated with the observable $\hat{M}=\hat{a}^{\dagger} \hat{b}+\hat{a} \hat{b}^{\dagger}$ 
displays two peaks for the ground state, that are the alive and dead state of the Schrödinger cat. This observable is associated with the population measurement in one of the two Zeeman levels. To observe the coherence we have to put the system in one of the two states, that corresponds to take a superposition of the ground and first excited state of the condensate. This can be obtained measuring with a nondemolition technique (e.g. a phase contrast technique) the population in a Zeeman level when only the ground state is populated. This observation collapses the $P(m)$ distribution to one peak, as discussed in Ref. 2. If the energy transfer is not too large we expect that only the first two energy states are populated. With this initial preparation the system begins to oscillate at the frequencies of Fig. 2 between the two Schrödinger cat states, as reported in Fig. 14. At the initial time only one peak is present (solid line). At a quarter of period corresponding to the frequency separation between ground and first excited state, the $P(m)$ displays two peaks (dashed line). At half a period the only peak is that absent at the initial time, thus there is a coherent oscillation between the two states. Detecting such an oscillation would provide evidence of a SC at an intermediate time when both peaks are present.

Notice that if we had $N$ loosely coupled or independent atoms ( $N g$ sufficiently low or even zero) the superposition of ground and first excited state would have a single peak, oscillating as a coherent state inside a harmonic potential. This would by no means be a SC. On the contrary, we have shown that, for $N g$ sufficiently high, we have a two peak distribution with the two partial barycenters at nearly constant positions. During the evolution, the two peak amplitudes oscillate, that is, the probability to find the system in either state oscillates.

In conclusion, we have shown that a double well dispersion curve can be obtained by a suitable Raman coupling. In this situation a symmetry breaking in momentum space is demonstrated solving two coupled GrossPitaevskii equations. The condensate can oscillate between the two emerging asymmetrical steady solutions (SC states) by means of the field quantum fluctuations (MQC). We have found that it is possible to obtain an oscillation frequency between the SC states around $50 \div 100 s^{-1}$. In order to neglect the thermal activation, the second excited level energy $\left(E_{2}\right)$ has to be higher than the thermal energy. From Fig. 2 we can see that $E_{2}$ ranges between $100 \div 300 \mathrm{~s}^{-1}$, that correspond to a temperature of $0.7 \div 2.3 n K$. If the cooling is performed below the threshold, when the symmetry breaking does not occur, the required temperature can be $\sim 5 n K$. However it may not be necessary to cool at very low temperatures the whole condensate, but just the involved degrees of freedom, provided that this latter ones are weakly coupled with the other modes, which act as a thermal bath. We remark that for low densities the evaporative cooling allows to reach much lower temperatures, because of a smaller three-body decay rate. To be sure that no excitation is present one could tailor the trapping potential in such a way that only the first two levels are bound. In this work we have chosen the parameters for which the tunneling frequency is much larger than the decoherence rate, however the symmetry breaking and the super-Poissonian atom fluctuations below threshold can be observed with a much higher number of atoms, thus these phenomena are observable with the present technology.
[1] M. Greiner et al., Nature 415, 39 (2002).

[2] A. Montina and F.T. Arecchi, Phys. Rev. A, in press. Scheduled issue: 1 July 2002; cond-mat/0202234.

[3] A. J. Leggett, Prog. Theor. Phys. Supplement 69, 1 (1980); A. J. Leggett, A. Garg, Phys. Rev. Lett. 54, 857 (1985).

[4] C. Monroe et al., Science 272, 1131 (1996).

[5] M. Brune et al., Phys. Rev. Lett. 77, 4887 (1996).

[6] J. I. Cirac et al., Phys. Rev. A 57, 1208 (1998).

[7] D. Gordon and C. M. Savage, Phys. Rev. A 59, 4623 (1999).

[8] A. Montina, F. T. Arecchi, Journ. Mod. Opt. 49, 319 (2002).

[9] C. J. Myatt et al., Phys. Rev. Lett. 78, 586 (1997).
[10] M. D. Barrett, J. Sauer, and M. S. Chapman, Phys. Rev. Lett. 87, 010404 (2001).

[11] B. P. Anderson and M. A. Kasevich, Science 282, 1686 (1998); D. M. Stamper-Kurn et al., Phys. Rev Lett. 80, 2027 (1998); H.-J. Miesner et al., Phys. Rev. Lett. 82, 2228 (1999); D. M. Stamper-Kurn et al., Phys. Rev. Lett. 83, 661 (1999).

[12] D. Gordon and C. M. Savage, Phys. Rev. A 58, 1440 (1998).

[13] E. A. Burt et al., Phys. Rev. Lett. 79, 337 (1997).

[14] D. Guéry-Odelin and G. V. Shlyapnikov, Phys. Rev. A 61, 013605 (2000). 